Document downloaded from:

http://hdl.handle.net/10251/43736

This paper must be cited as:

García Pineda, M.; Lloret, J.; Sendra Compte, S.; Rodrigues, JJPC. (2011). Taking Cooperative Decisions in Group-Based Wireless Sensor Networks. En Lecture Notes in Computer Science. Springer Verlag (Germany). 61-65. doi:10.1007/978-3-642-23734-8_9.

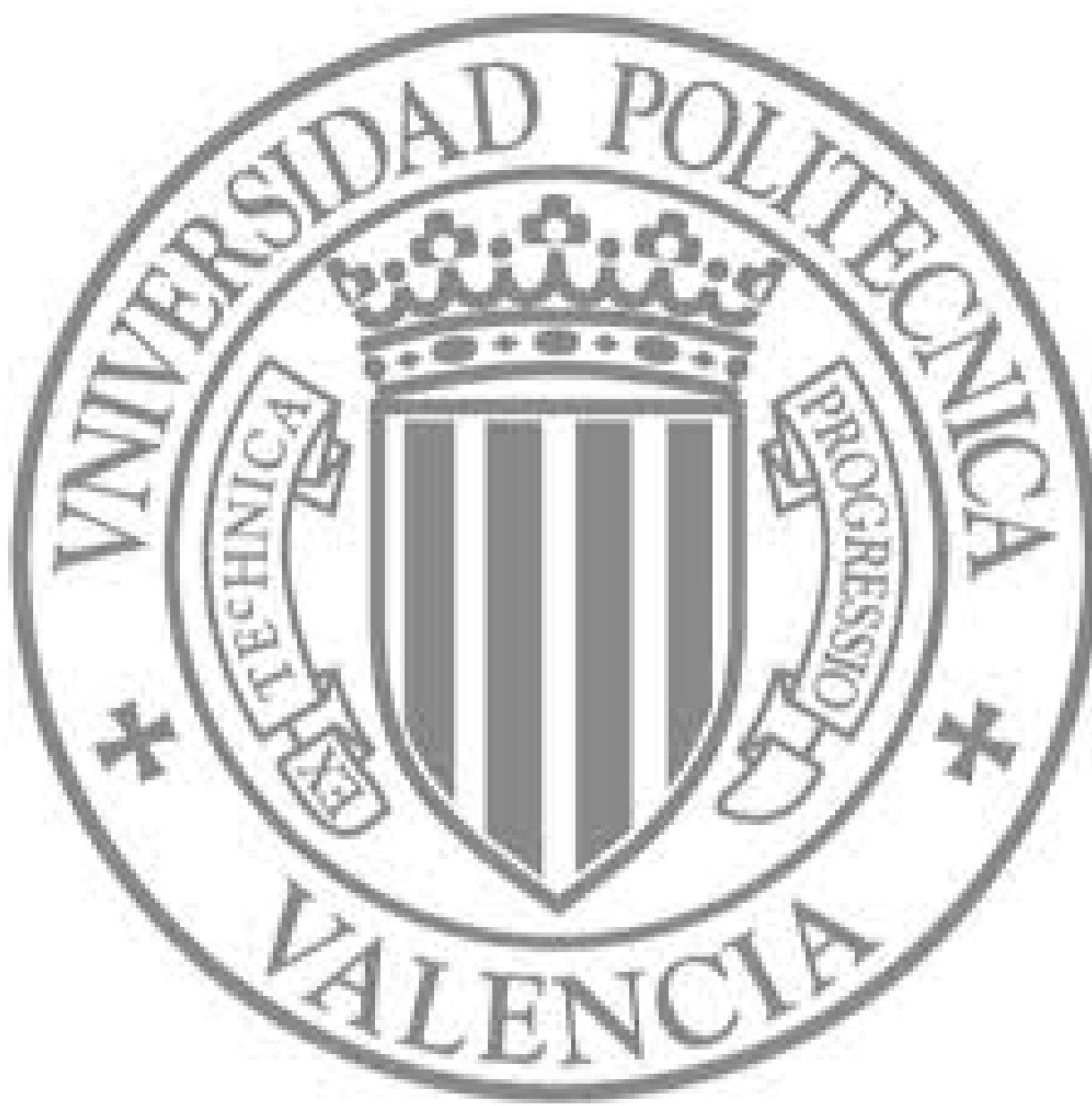

The final publication is available at

http://dx.doi.org/10.1007/978-3-642-23734-8_9

Copyright Springer Verlag (Germany) 


\title{
Taking Cooperative Decisions in Group-Based Wireless Sensor Networks
}

\author{
Miguel Garcia ${ }^{1}$, Jaime Lloret $^{1}$, Sandra Sendra ${ }^{1}$, Joel J. P. C. Rodrigues ${ }^{2}$ \\ ${ }^{1}$ Integrated Management Coastal Research Institute. \\ Universitat Politècnica de Valencia. Camino de Vera s/n, 46022 Valencia, Spain \\ ${ }^{2}$ Instituto de Telecomunicações. \\ University of Beira Interior, Av. Marquês D'Ávila e Bolama, 6201-001 Covilhã, Portugal \\ migarpi@posgrado.upv.es, jlloret@dcom.upv.es, sansenco@posgrado.upv.es, joeljr@ieee.org
}

\begin{abstract}
Several studies have demonstrated that communications are more efficient when cooperative group-based architectures are used in wireless sensor networks (WSN). This type of architecture allows increasing sensor nodes' lifetime by decreasing the number of messages in network. But, the main gap is to know how to take cooperative decisions in order to make the right communication. In this paper, we analyze the main aspects related to collaborative decisions in WSNs. A mathematical analysis will be presented in order to take the correct decision. Finally, the simulations will show the efficiency of the method used to make cooperative decisions in WSNs.
\end{abstract}

Keywords: cooperative decisions, cooperative WSN, group-based WSN.

\section{Introduction and Related Work}

Nowadays, there are a lot applications based on WSNs [1]. Most of them are based on centralized or distributed architectures, but we proved in a previous work that groupbased architectures are more efficient in terms of performance and energy [2]. Moreover, we proved the benefits of a cooperative group-based WSN for environmental monitoring in [3]. In this case, when a group detects an event, it warns the alert, jointly with the parameters measured, to its neighboring groups. Cooperation with other groups could change the direction of the alert propagation and the level of the alert. According to this cooperation, the sensor network was efficient and the sensors had a longer lifetime [4]. But, how the sensor nodes take the cooperative decisions? There are some works, where the authors talk about the process to take the correct cooperative decision. For example, in [5], the authors propose a new approach to estimate the credibility of decision makers in a knowledge grid environment. They developed a new fuzzy operator, which aggregates decisions made inside the decision makers' community and considering the credibility of the decision makers.

In [6], there is another work where authors explain how to make distributed decisions in cooperative systems. They define cooperation processes and a set of models that are able to support designers of cooperative decisions. These models come from a more general architecture of a Cooperative Knowledge Based System and are based on the knowledge acquisition field. Finally, a paper which revises the 
group decision over cooperative work is shown in [7]. The authors review the group decision support systems (GDSSs) that have been configured to meet the needs of groups at work, and they evaluate the experience to date with such systems.

There are more papers with systems taking collaborative decisions, but none of them are designed for WSNs. Moreover, there is not any system for cooperative group-based WSNs where the decision is agreed with their neighbors based on their sensed parameters. In this paper, we assume that the energy consumed by processing tasks is lower than the power consumed in transmission [4], so a collaborative decision system will allow saving energy in the WSN.

The rest of the paper is organized as follows. In section 2, the mathematical analysis used for taking cooperative decisions in group-based WSNs is described. In Section 3, the simulation that shows the efficiency of the decision system is provided. Finally, in Section 4, we draw the conclusion.

\section{Cooperative Decision}

The cooperative decision system described in this section has been designed for the cooperative group-based WSN for environmental monitoring presented in [3]. In this system, the network is divided into groups, in which each group is formed by several sensor nodes. All sensor nodes sense several parameters. The parameters sensed by each node and the cooperation between groups make an efficient monitoring network. During the observation time, each sensor node gathers 4 parameters: temperature of the environment $T=\left\{t_{0}, t_{1}, t_{2}, t_{3}, \ldots, t_{n}\right\}$, humidity is $H=\left\{h_{0}, h_{1}, h_{2}, h_{3}, \ldots, h_{10}\right\}$, wind $W=\{$ speed $, x, y, z\}$ and fire $F=\left\{f_{\text {yes }}, f_{\text {no }}\right\}$. Where $\mathrm{T}$ can be in Celsius, $\mathrm{H}$ is humidity in $\%$, the wind variable, formed by its speed and the direction, and, finally, the fire variable, which gives the presence of fire. According to these parameters we define 6 network states (see Table 1).

Table 1. Possible states of our cooperative group-based WSN.

\begin{tabular}{ll}
\hline State & Definition \\
\hline S0 & No fire + No wind + High humidity $\geq \mathrm{h}_{5}+$ Low temperature \\
S1 & No fire + No wind + Low humidity $\leq \mathrm{h}_{5}+$ High temperature \\
S2 & No fire + Wind + Low $/$ high humidity + High temperature \\
S3 & Fire + No wind + High humidity $\geq \mathrm{h}_{5}+$ Low temperature \\
S4 & Fire + No wind + Low humidity $\leq \mathrm{h}_{5}+$ High temperature \\
S5 & Fire + Wind + Low/high humidity + High temperature \\
\hline
\end{tabular}

We have also defined 6 actions that can be performed by the network in each state. When the network is idle, action $A O$ happens (no alert). When there is low humidity and high temperature $(A 1)$, level 1 alert is sent to the same group. Action $A 2$ will send the level 1 alert to the same group and to neighboring groups in the same direction of the wind. $A 3$ sends the level 2 alert to the same group and this action is taken when there is a fire. $A 4$ sends the level 3 alert to the same group and this action is taken when there is a fire, wind, low humidity, and high temperature. Finally, $A 5$ sends the level 3 alert to the same group and to all neighboring groups, and this action happens when is there is a fire, wind, low humidity and high temperature. 
Each state has an adequate action. The network must be able to choose the most appropriate action according to its state and on what is happening. In order to do this process, we used a decision system which is based on the uncertainty. The decision maker knows the possible states, but it does not have any information about which of them is the best state to be changed to. Not only it is unable to predict the next state, but also it cannot quantify in any way this uncertainty. In particular, this excludes the knowledge of probabilistic information on the possibilities of occurrence of each state. In order to develop the decision criterion it has to know the matrix of criteria (see Fig. 1a), where in each box is defined by the probability of performing an action for a state. It will be performed by each node, e.g. brown node in Fig. $1 \mathrm{~b}$.

\begin{tabular}{|c|c|c|c|c|c|c|c|}
\hline & \multicolumn{6}{|c|}{ States } \\
\hline & & So & $S 1$ & $S 2$ & S3 & $S 4$ & S5 \\
\hline \multirow{6}{*}{$\begin{array}{c}\text { Action } \\
\mathbf{s}\end{array}$} & $A 0$ & $\mathrm{x}_{0,0}$ & $\mathrm{X}_{0,1}$ & $\mathrm{X}_{0,2}$ & $\mathrm{x}_{0,3}$ & $\mathrm{X}_{0,4}$ & $\mathrm{x}_{0,5}$ \\
\hline & $A 1$ & $\mathrm{x}_{1,0}$ & $\mathrm{x}_{1,1}$ & $\mathrm{X}_{, 2}$ & $\mathrm{x}_{1,3}$ & $\mathrm{x}_{1,4}$ & $\mathrm{X}_{1,5}$ \\
\hline & $A 2$ & $\mathrm{X}_{2,0}$ & $\mathrm{x}_{2,1}$ & $\mathrm{X}_{2,2}$ & $\mathrm{x}_{2,3}$ & $\mathrm{X}_{2,4}$ & $\mathrm{X}_{2,5}$ \\
\hline & $A 3$ & $\mathrm{x}_{3,0}$ & $\mathrm{X}_{3,1}$ & $\mathrm{X}_{3,2}$ & $\mathrm{x}_{3,3}$ & $\mathrm{X}_{3,4}$ & $\mathrm{X}_{3,5}$ \\
\hline & $A 4$ & $\mathrm{X}_{4,0}$ & $\mathrm{X}_{4,1}$ & $\mathrm{X}_{4,2}$ & $\mathrm{X}_{4,3}$ & $\mathrm{X}_{4,4}$ & $\mathrm{X}_{4,5}$ \\
\hline & $A 5$ & $\mathrm{X}_{5,0}$ & $\mathrm{X}_{5,1}$ & $\mathrm{X}_{5,2}$ & $\mathrm{X}_{5,3}$ & $\mathrm{X}_{5,4}$ & $\mathrm{X}_{5,5}$ \\
\hline
\end{tabular}

Fig. 1a. Matrix of criteria.

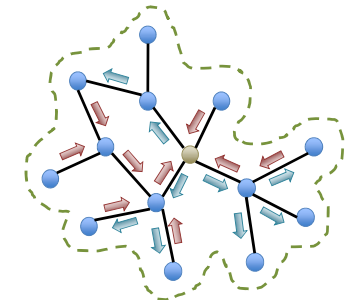

Fig. 2b. A group of our proposed WSN.

The matrix of criteria is based on the criterion of Savage [8], which indicates that the decision maker compares the result of an action under a state with all other outcomes, regardless of the state under which they occur. However, the state is not controllable by the decision maker, so that the result of an action should only be compared with the results of the other alternatives under the same state of nature. For this purpose, Savage defines relative loss or loss of opportunity $r_{i, j}$ (see equation 1) associated with a result $x_{i, j}$ as the difference between the result of the best alternative because $S i$ is the true state and outcome of the action $A_{i}$ under the state $S_{i}$.

$$
r_{i, j}=\max _{1 \leq k \leq m}\left\{x_{k, j}\right\}-x_{i, j}
$$

But the Savage criterion propose to select the action that provides the smallest of the major losses suffered $\left(\rho_{i}\right)$, i.e., if $r_{i}$ is defined as the greatest loss that can be obtained by selecting the action $A_{i}$ (see equation 2 ).

$$
\rho_{i}=\max _{1 \leq j \leq n}\left\{r_{i, j}\right\}
$$

For the application of this criterion, the node calculates the matrix of relative losses which consists of the $r_{i, j}$ elements. Each column of this matrix is obtained by calculating the difference between the maximum value of that column and each one of the values listed. For selecting the best action in each state we use the equation 3 .

$$
r\left(A_{i}, S_{j}\right)=\left\{\begin{array}{cc}
\max _{a_{k}}\left\{x\left(A_{k}, S_{j}\right)-x\left(A_{i}, S_{j}\right)\right\} & \text { Good decision } \\
x\left(A_{i}, S_{j}\right)-\min \left\{x\left(A_{k}, S_{j}\right)\right\} & \text { Bad decision }
\end{array}\right\}
$$

\section{Simulation of the cooperative decision system}

In order to evaluate the accuracy of our cooperative decision system we simulated it. We took the example provided in Fig 1b. This simulation has been done using 
Matlab. In each case when an event happens in a sensor node, it will send an information request to its neighbors, which will reply with their information and, after taking a decision based on the information received, it will reply with the decided action. Figure 2a shows the protocol procedure. We have defined 20 possible cases making a matrix of criteria. Then, we applied our decision system based on the Savage criterion during 100 times. Fig. $2 \mathrm{~b}$ shows the estimated average for each case. In this figure we can see the action selected by each state. The best solution in each state is the action with the same subscript, i.e., $S_{n} \rightarrow A_{n}$. We can see in this Fig $2 \mathrm{~b}$ that our system is not perfect, but it only has an error of $3.52 \%$.

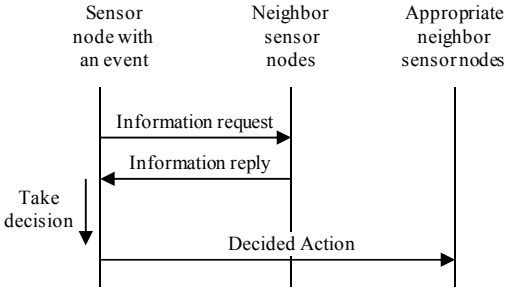

Fig. 3a. Protocol procedure.

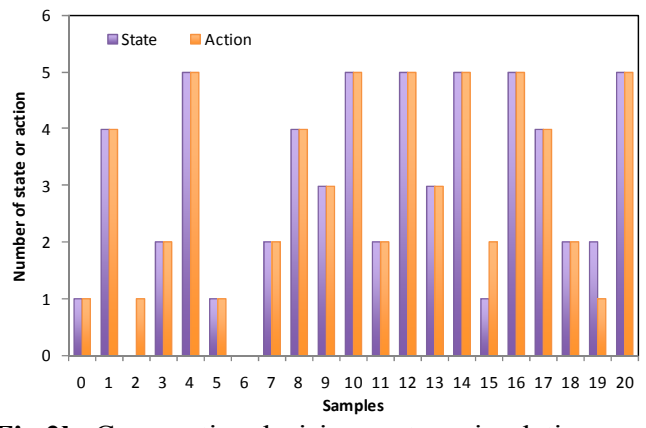

Fig.2b. Cooperative decision system simulation.

\section{Conclusion}

In this paper we propose a cooperative decision system based on several parameters for selecting the better action in a group-based WSN for environmental monitoring. According to this decision, the sensor node sends the appropriate level alert to its group or to the appropriate neighboring groups. Simulation shows that the accuracy of the criterion is quite good because the system only has an error of $3.52 \%$.

\section{References}

1. Garcia, M., Bri, D., Sendra, S. and Lloret, J.: Practical Deployments of Wireless Sensor Networks: a Survey. Int. Journal on Advances in Networks and Services, vol. 3, no. 3\&4, pp. 170-185 (2010).

2. Lloret, J., Garcia M. and Tomas, J.: Improving Mobile and Ad-hoc Networks performance using GroupBased Topologies. Wireless Sensor and Actor Networks II. IFIP International Federation for Information Processing, Vol. 264/2008, pp. 209-220 (2008).

3. Garcia, M. and Lloret, J.: A Cooperative Group-Based Sensor Network for Environmental Monitoring. Cooperative Design, Visualization, and Engineering 2009, LNCS, Vol. 5738/2009, pp. 276-279 (2009).

4. Garcia, M., Sendra, S., Lloret, J. and Lacuesta, R.: Saving Energy with Cooperative Group-Based Wireless Sensor Networks. CDVE 2010. LNCS, Vol. 6240/2010, pp. 73-76 (2010).

5. Parsa, S. and Parand, F-A.: Cooperative decision making in a knowledge grid environment. Future Generation Computer Systems, vol. 23, pp. 932-938 (2007).

6. Soubie, J.-L. and Zaraté, P. Distributed Decision Making: A Proposal of Support Through Cooperative Systems. J. Group Decisions and Negotiation, Vol. 14, Num. 2, pp. 147-158 (2005).

7. Kraemer, K. L. and King, J. L. Computer-based systems for cooperative work and group decision making. ACM Computer Survey. Vol. 20, Num. 2, pp. 115-146 (July 1988).

8. Kernan, J. B. Choice Criteria, Decision Behavior, and Personality. Journal of Marketing Research, vol. 5, no. 2, pp. 155-164 (May 1968). 